\title{
Pathway to cost-efficient state estimation of future distribution networks
}

DOI:

10.1109/PESGM.2016.7741337

\section{Document Version}

Accepted author manuscript

Link to publication record in Manchester Research Explorer

\section{Citation for published version (APA):}

Liao, H., \& Milanovic, J. V. (2016). Pathway to cost-efficient state estimation of future distribution networks. In Power and Energy Society General Meeting (PESGM), 2016 https://doi.org/10.1109/PESGM.2016.7741337

\section{Published in:}

Power and Energy Society General Meeting (PESGM), 2016

\section{Citing this paper}

Please note that where the full-text provided on Manchester Research Explorer is the Author Accepted Manuscript or Proof version this may differ from the final Published version. If citing, it is advised that you check and use the publisher's definitive version.

\section{General rights}

Copyright and moral rights for the publications made accessible in the Research Explorer are retained by the authors and/or other copyright owners and it is a condition of accessing publications that users recognise and abide by the legal requirements associated with these rights.

\section{Takedown policy}

If you believe that this document breaches copyright please refer to the University of Manchester's Takedown Procedures [http://man.ac.uk/04Y6Bo] or contact uml.scholarlycommunications@manchester.ac.uk providing relevant details, so we can investigate your claim.

\section{OPEN ACCESS}




\title{
Pathway to Cost-Efficient State Estimation of Future Distribution Networks
}

\author{
Huilian Liao, Member, IEEE and Jovica V. Milanović, Fellow, IEEE \\ School of Electrical and Electronic Engineering, \\ The University of Manchester, Manchester, UK \\ milanovic@manchester.ac.uk
}

\begin{abstract}
This paper provides a detailed overview of the techniques and applications related to distribution system state estimation (DSSE), together with the classification of various types of state estimation. The paper also provides the state-of-art techniques applied in DSSE including forecasted-aided state estimation, close-loop DSSE methods, the application of computation intelligence in DSSE and the use of smart meters and phasor measurement units in load estimation. As more and more active elements and functionalities will be integrated in future distribution network, e.g. demand-side management, the increased penetration of decentralized generation and dynamically controlled storage/devices, DSSE will be one of the critical functionalities for secure operation of future distribution networks. As a pathway to cost-efficient DSSE for future distribution networks, this paper addresses the characteristics of future distribution grids that will affect DSSE, and also discusses the techniques and data that can be used to better understand/model the network behaviors and load profiles in order to ultimately improve the accuracy of DSSE.
\end{abstract}

Index Terms-State estimation, smart meters, phasor measurement units, computation intelligence, load estimation.

\section{INTRODUCTION}

Secure operation of power systems requires a comprehensive understanding of the system operating status, which helps in identifying potential critical operating conditions and determining the necessary preventative measures. Power system state estimation (SE) plays an important role in secure operation of power systems [1]. It provides an optimal estimate of the system states based on both continuous monitored measurements and pseudomeasurements. The concern of state estimation was first addressed in 1970's [2], and later with the increasing capability of SCADA system computers and establishment of Energy Management Systems (EMS), this functionality was widely integrated in EMS and used for operation and management of transmission systems [3].

With the increasing penetration of distributed renewable energy resources, the connection of medium-sized distributed generation (DG) and more and more electronic interfaced devices (e.g., different types of load storage and electric vehicles) connected in the distribution networks, it is required to have an improved observability of the distribution networks in order to ensure secure operation of distribution systems. This led to the intensive research and application of SE at distribution levels, namely Distribution System State Estimation (DSSE).

Different from meshed transmission networks, most distribution networks are radial, often with high $\mathrm{R} / \mathrm{X}$ ratios. Besides, application of real time measurement is very limited in distribution networks. The SE approaches developed for transmission networks such as decoupled methods and DC approximations cannot be applied to DSSE directly [4]. Furthermore, ill conditioned matrices and large number of nodes in distribution networks imposes great difficulty to DSSE. Different from transmission system SE which is to improve the reliability of the measured values, the usual purpose of DSSE is to extend the observability of the network. With the rigorous requirement of observability in distribution networks, the development of DSSE has attracted great attention recently. Various techniques have been investigated for DSSE in literature, such as Weighted Least Squares (WLS) based SE [1], fuzzy SE [5] and branch-based SE [6], and so on. Besides, various types of data in distribution networks including Advanced Metering Infrastructure data have been extensively explored for the purpose of DSSE [4, 7-13].

Future distribution networks will evolve into more flexible and automatically controlled networks with increasing "selffunctionalities". It is anticipated that more and more decentralized generation units (e.g., micro-CHP, solar panels and wind turbines) will be connected to the grid, and increased power will be injected at lower voltage levels. Furthermore, various stakeholder actors will take increased active involvement in network operation and control. Complicated interaction/collaboration among actors, e.g., the exchange of flexibility and services and demand side management, will change the grid configuration and load profiles frequently. To facilitate this, accurate state estimate of the network status will become unprecedentedly important. This paper addresses the characteristics of future distribution networks and discusses the techniques and data that can be used to develop accurate and efficient DSSE for future distribution networks.

\section{State ESTIMATION (SE)}

\section{A. Description of SE Processes}

$\mathrm{SE}$ processes are illustrated in Figure 1. First, topology processor verifies the up-to-date network parameters and configures the one-line diagram of the network; observability analysis tool identifies the un-observed branches in the 
network and establishes the required but missing information, often referred to as pseudo measurements; given all available input data including topology, measurements and pseudo measurements, the state estimator searches for an optimal estimate of the system state; bad data processor identifies and eliminates the data affected by gross errors and noise. To continuously update and improve the pseudo measurements, the estimated results can also be used as feedback to adjust the methodologies of load estimation and load forecasting, and form a "close-loop" information flow.

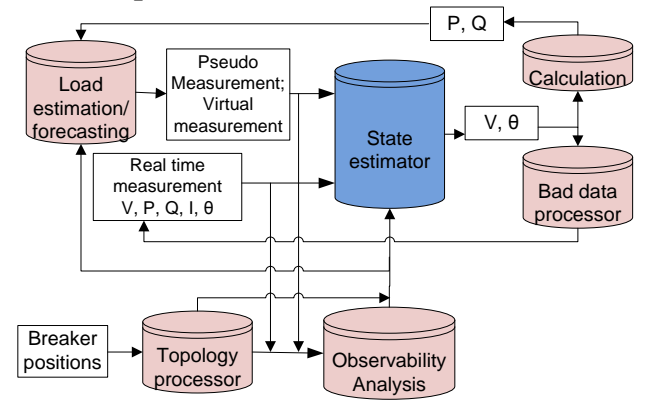

Figure 1. Illustration of SE processes.

\section{B. Classification of SE and Application}

Depending on the timing and evolution of the estimation, SE schemes can be classified into three categories:

1) Static $S E$ - static $\mathrm{SE}$ is a data processing algorithm which converts measurements and other available information into an estimate of the state of an electric power system. For static SE, the measured data are taken to be time invariant. It relies on a single set of measurements all taken at one snapshot in time. Various methods have been investigated for static DSSE, and the most common approach to state estimator is WLS method [1].

2) Tracking $S E$ - tracking SE utilizes the recent available states of the network to update the network state noniteratively (usually using one iteration) for the subsequent sampling period. It calculates the error between the new measurements and recent estimated values, and then uses it as "feed-back error signal" to correct the old estimate and obtain the new estimates via a gain matrix [14]. Tracking SE has the merits of efficient computation and being suitable for realtime implementation.

3) Dynamic $S E$ - dynamic SE is also named as ForecastAided SE (FASE). To avoid confusion with true dynamic SE (the transients in power systems) that usually occurs at a much faster time scale, the abbreviation of FASE is used in this paper. FASE utilizes, in addition to the present states, the previous estimates of the states to perform one step ahead SE [15-19]. A short-term forecast (e.g. several seconds/minutes ahead) of the state variables is made, and each time when a new set of measurements becomes available, an "innovation analysis" is used to determine if the new measurements are significantly different from the predicted values. In FASE, dynamic model for the time behavior of system states is utilized, whereas tracking and static SEs do not require any dynamic model of the system states. FASE has the advantage of being able to forecast the state vector one step ahead. Furthermore, the knowledge of the time-evolution of the state can be also used as extra information to make a better estimation of current state. Although most FASE techniques and applications proposed to date are focused at the transmission level, FASE approaches are attracting increased attention in DSSE.

\section{Power Quality SE}

Recently, the increased awareness of power quality (PQ) issues in distribution networks has led to more research focused on PQ state estimation (PQSE). PQSE covers different types of analysis in PQ area. Figure 2 lists four types of PQSE [20], together with the purposes of their application.

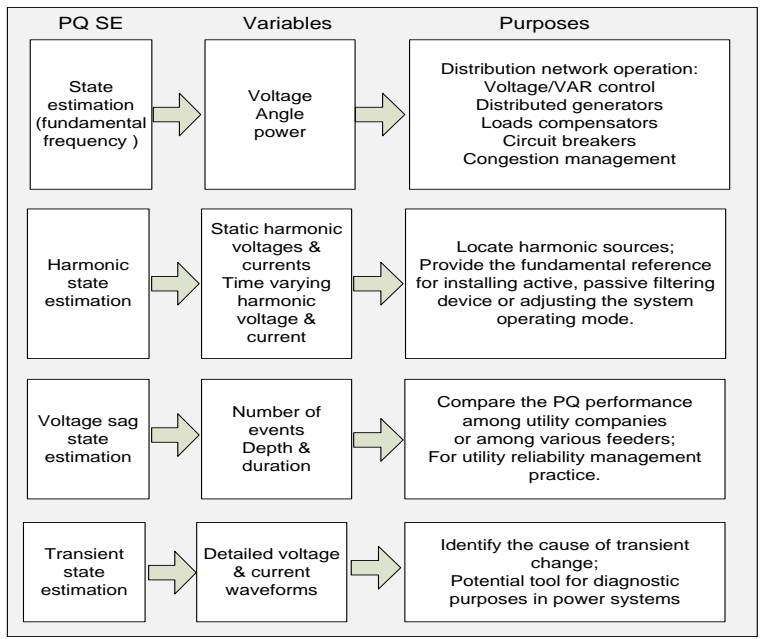

Figure 2. Classification of PQSE.

1) General $S E$ - general SE is performed at fundamental frequency in order to estimate the network state represented by voltage, angle and power, etc. The estimated results can be used for distribution network operation and control, e.g., voltage regulation, load compensator, congestion management, phase imbalance correction and reactive power compensation [21], etc. The performance of voltage unbalance at all buses can also be estimated using general SE through postprocessing the estimated three-phase voltages [22-24].

2) Harmonic state estimation (HSE) - HSE generates the "best" estimate of the harmonic levels from limited measured harmonic data [25], in order to locate harmonic sources and estimate harmonic distribution. It provides the fundamental reference for installing active, passive filtering device or adjusting the system operating mode, to reduce system harmonic levels and hazards and ensure the safe operation of the System [26].

3) Voltage Sag SE (VSSE) - VSSE estimates sag characteristics at unmetered nodes, including the number of voltage sags and sag profiles/levels. This concept is borrowed from utility reliability management practice [27], and driven by the need to compare the PQ performance among utility companies or among various feeders. Various techniques have been explored for VSSE in literature, e.g., fault location, probability based methods and Monte Carlo methods [28, 29].

4) Transient SE (TSE) - TSE estimates the instantaneous voltages and currents at unmetered nodes in the distribution networks. It can be exploited to identify the cause of transient change in system parameters and can be a potential valuable tool for diagnostic purposes in power systems [30, 31]. 


\section{Uncertainty and Estimation Accuracy}

The deviation/uncertainty of input data/information impacts the accuracy of DSSE to a certain extent.

1) Measurement uncertainty: DSSE works with a limited number of real measurements. Measurement error and bias exist in each measurement. The measurement can be characterized by its own range of measurement errors which is primarily determined by the corresponding measurement devices [32]. Due to the lack of real measurements, pseudomeasurements play an important part in DSSE. The deviation of both real and pseudo- measurements can greatly affect the accuracy of system state estimation. A number of dedicated papers provide the analyses regarding the impact of measurement uncertainty (pseudo-measurements and heterogeneous measurements) on SE accuracy [33-35]. Reference [36] analyses the relationship between the measurement errors existing in both load pseudo measurements and real-time measurements and the deviations of the system state estimation, presenting how increasing the load measurement accuracy will improve the estimated deviations of bus voltages. In [35], the impact of different types of measurements and the accuracies of the measurement devices on accuracy of DSSE is analysed. In [37], the classic WLS based SE is analysed to point out under which circumstance and to what extent SE results are affected by measurement uncertainty when a minimum number of measurements which is just enough to assure full observability is used. It provides a straightforward criterion to predict the overall worst-case SE accuracy or, dually, to establish the maximum measurement uncertainty that is able to keep the average or worst-case estimation accuracy within given boundaries.

2) Uncertainty of operating conditions: The impact of the uncertainty of the network behavior (such as distributed voltage control and demand side management) on state estimation is analysed in [35], which demonstrates that investment in the improvement of the knowledge of the distribution grid behavior is inevitable in order to improve the accuracy of DSSE.

3) Network parameters uncertainty: Network parameter uncertainty would also impact the accuracy of DSSE. The impact of network parameters uncertainty on state estimator's bias is analysed in [38], and the tolerance of the value of the network parameters is analysed by means of suitable Monte Carlo procedures in [39].

4) Topology uncertainty: In distribution networks, the network topology in operation can change very frequently. For instance, switching is usually used as a resource to improve system operation or to reduce the impact of outages. The frequent topology changes in distribution networks together with the reduced number of installed measurement devices can lead to situations in which the topology is not known beyond any doubt even after running the topology processor. The impact of topology uncertainty on estimation accuracy is analysed in [40]. In [41], the network configuration changes are identified using recursive Bayesian approach by utilizing the outputs of state estimation function.

5) Input data correlation: Correlation usually exists among different input data. For instance, a degree of correlation can often be assumed among power consumptions or generations of some particular nodes. The impact of parameters correlation on the quality of the estimation is analysed in [38], and the influence of input data correlation on DSSE is investigated in [42, 43].

\section{STATE OF THE ART}

This section briefly describes some of the most advanced techniques and applications of DSSE in literature.

\section{A. Load Estimation}

In distribution networks, the lack of real measurements is compensated by the use of information from the loads. Thus a high proportion of the measurements can be pseudomeasurements. Load estimation (or pseudo-measurements) plays an important role in DSSE. Computation intelligence methods have been investigated for DSSE. For instance, artificial neural network has been adopted to generate pseudo measurements based on a few real measurements in conjunction with typical load profiles [44]. In [45], machine learning methodologies have been investigated to provide reliable input information to a robust state estimation algorithm. Real time estimation of loads can also be obtained based on iterating between WLS estimator and load flow [46].

\section{B. Use of Measurements}

More comprehensive and accurate knowledge of the system is required in order to make efficient and reliable control actions. In particular, attention should be paid to phase angles estimation which can be used to avoid critical situations. In this context, the use of phasor measurement units (PMU), which provides synchronized local measurement with global time stamp, is promising. PMUs measure not only voltage phasors but also current phasors through all incident buses. With the increasing deployment of PMUs in the distribution grids, state-of-the-art SE are implemented using data from PMUs [7, 8]. The benefits of the use of PMU data and the approach of integrating PMU data in DSSE have been extensively investigated in literature [43, 47].

The roll-out of smart meters means that an unprecedented amount of detailed historical data on user loads is becoming available. This data can be used to better understand and model the behaviors of distribution network loads, allowing to improve load estimation techniques, and ultimately, DSSE accuracy [4]. Studies have been made into the incorporation of smart meter data in DSSE to estimate flows, voltages, and losses in low voltage distribution networks [9-12].

\section{State Estimator}

Various advanced computation intelligence techniques have been extensively explored for DSSE. Auto-associative neural networks (autoencoders), which only require historical database and few quasi-real-time measurements to perform an effective SE, are used to learn the behavior of the grid and avoid the need of characterizing the grid parameters and topology [48]. Hybrid particle swarm optimization was adopted to estimate bus voltage magnitudes and angles, and discrete tap values of on-load tap changers [49]. In [45], machine learning methodology collaborating with SE forms a closed-loop information flow in order to continuously update and improve the performance of the state estimator. 
Since distribution systems are typically very large and dense, to improve the calculation efficiency, distributed SE (also named multi-area) is investigated for processing separate sub-systems in parallel, in conjunction with a certain level of coordination or agent communication [7, 50, 51]. In order to accomplish very large-scale monitoring of interconnected power systems, multi-level, or hierarchical SEs, are also developed to integrate existing SEs that are designed to function at different levels of modeling hierarchy [52]. These distributed SEs can greatly enhance the computational performance and improve the reliability of SE algorithms.

To allow the system operators to have more time in making control decisions, FASE approaches are attracting more and more attention in DSSE, particularly when highresolution data from synchronised metering devices such as PMUs are available. To make a more accurate mathematical description of the time evolution of the state, attempts have been made to generate a more accurate mathematical description of the time evolution of the state using Kalman filter and other techniques [7, 53, 54].

\section{DSSE OF FUTURE DISTRIBUTION NETWORKS}

Future distribution networks will be characterised by an unprecedented integration of large-scale Renewable Energy Sources, a wide range of electricity generating technologies, various power electronics-interfaced storage devices, various dynamically controlled elements and different formats of demand response schemas. The networks will be engaged with a high level of active involvement from various actors (e.g., DSO, retailers, ESCOs, aggregators, customers, industrial and domestic prosumers) and will be highly affected by electricity market which will be more flexible and has more participants. The active actors will be able to access huge data streams and interface with different parts of the networks. They will be actively involved in electricity markets and manage their services/assets/loads dynamically in real time to improve operating efficiency and reduce cost. In summary, the future distribution networks will be much more flexible, smarter and more active compared to conventional passive distribution networks. The operation of future distribution networks faces the toughest challenge due to the randomness and uncertainties of the grid behaviors, e.g., the uncertain outputs of scattered distributed generators and the unpredictable behaviors of various actors, etc. Development of DSSE that addresses these new features is becoming more stringent than ever in terms of future network operation and control.

The future networks will be equipped with a significant increase and reliance on monitoring and processing of large amount of data streams. More and more data/information will be provided in different formats with different frequencies for different purposes. More PMUs will be deployed in future power grids, thus more phasor information will be available to make DSSE more dynamic and reactive to local disturbances before effects cascade through the system. Due to the high cost of PMU installation, PMU deployment in the distribution networks will continue to be selective for the foreseeable future. Other available sources of data/information should also be explored for DSSE, such as the massive amount of data coming from smart meters. DSSE will also take advantage of all other available data which could be originally used for other purposes (e.g., operation, pricing and control) in the networks, such as billing data, electricity market information, actors' profiles, actors' preference, flexibility service provided by actors and demand response scheme, etc. These data can be exploited and processed to extract useful information that can be used to understand and model the behaviors of distribution networks, improving load estimation and the estimation of the status of the network equipment/devices such as dynamically controlled storages and electric vehicles. This will also contribute to the identification of network configuration changes and network topology analysis, and ultimately, DSSE accuracy. Besides, instead of deploying dedicated data retrieving systems for DSSE purpose only, taking advantage of all available resources without needing additional specific devices in the grids is a cost-effective pathway for future DSSE development. In this context, methodologies of big data analyst (e.g., data and text mining approaches) which model/construct the correlation among different parameters will be required to convert data/text into information and transform it into actionable intelligence. The integration of big data techniques in DSSE (for load estimation and topology analysis) will be extensively investigated for SE purposes.

On the other hand, when various types of data are shared and become available, there is a risk that the grid's operator will be drowned in data. To facilitate huge data streams, communication infrastructure with large capacity is required throughout the grid, which suggests that a high cost of communication investment is required. To avoid this, an event-triggered approach for sensing, communicating and information processing would be quite appealing. The challenge here is to provide analytical performance guarantees in a distributed event triggering algorithm in a dynamically changing environment. In the event-triggered SE, the state estimates are updated only when the measurements received indicate that there is a potential issue $[3,4]$.

Most SE methods proposed to date in literature have the feature of using open-loop information flow. However, as the network becomes more complicated, a closed-loop DSSE is becoming more appealing, due to that it allows the predictive database to be continuously updated and improved based on feedback from the SE. In this way, loads, DG outputs and network configuration can be estimated more accurately and reliably. However, constructing the closed-loop information flow properly is challenging. More research is still required in developing DSSE with close-loop information flow. More computation intelligence methods will be extensively investigated for future DSSE development, in order to allow DSSE to be more intelligent and adapt to network changes spontaneously and quickly [4].

\section{CONCLUSIONS}

This paper provides a detailed overview of the techniques and application related to DSSE. Future distribution networks will be very different from present networks in terms of operation and active level of actors' involvement. This paper provides a pathway to cost-efficient DSSE by taking advantage of the available resources of future distribution networks, e.g., big data collected for various purposes in the network. Useful information will be extracted from all available information resources and used to understand the 
behaviors of the network elements (e.g., equipment/devices, loads and actors), and ultimately contribute to load estimation and topology analysis for DSSE. This paper also addresses the efficiency issue of processing a large amount of data, and discusses the potential solution, i.e. event-triggered SE. Finally, the paper also discusses the potential techniques to improve the performance of DSSE by focusing on close-loop DSSE and application of computation intelligence for the development of intelligent and dynamic DSSE.

\section{REFERENCES}

[1] A. Abur and A. G. Exposito, Power System State Estimation: Theory and Implementation. New York: Marcel Dekker, 2004.

[2] F. C. Schweppe and J. Wildes, "Power system static-state estimation, part I: exact model," IEEE Trans. Power Appar. and Syst., vol. PAS-89, pp. 120-125, 1970.

[3] H. Yih-Fang, S. Werner, H. Jing, N. Kashyap, and V. Gupta, "State estimation in electric power grids: meeting new challenges presented by the requirements of the future grid," IEEE Signal Proc. Mag., vol. 29, pp. 33-43, 2012.

[4] B. Hayes and M. Prodanovic, "State estimation techniques for electric power distribution systems," in Proc. Euro. Mode. Symp., 2014, pp. 303-308.

[5] A. T. Saric and R. M. Ciric, "Integrated fuzzy state estimation and load flow analysis in distribution networks," IEEE Trans Power Del., vol. 18, pp. 571-578, 2003.

[6] D. Youman, H. Ying, and Z. Boming, "A branch-estimation-based state estimation method for radial distribution systems," IEEE Trans Power Del., vol. 17, pp. 10571062, 2002.

[7] F. Baalbergen, M. Gibescu, and L. van der Sluis, "Modern state estimation methods in power systems," in Proc. Power Syst. Conf. Expo., 2009, pp. 1-6.

[8] M. Pau, P. A. Pegoraro, and S. Sulis, "Efficient branch-current-based distribution system state estimation including synchronized measurements," IEEE Trans Instrum Measure., vol. 62, pp. 2419-2429, 2013.

[9] R. F. Arritt, R. C. Dugan, R. W. Uluski, and T. F. Weaver, "Investigation load estimation methods with the use of AMI metering for distribution system analysis," in Proc. IEEE Rural Electric Power Conf., 2012, pp. B3-1-B3-9.

[10] A. Alimardani, F. Therrien, D. Atanackovic, J. Jatskevich, and E. Vaahedi, "Distribution system state estimation based on nonsynchronized smart meters," IEEE Trans Smart Grid, vol. 6, pp. 2919-2928, 2015.

[11] G. Lopez, J. Moreno, H. Amaras, and F. Salazar, "Paving the road toward smart grids through large-scale advanced metering infrastructures," Elec. Power Syst. Resear., 2014.

[12] S. Alam, B. Natarajan, and A. Pahwa, "Distribution grid state estimation from compressed measurements," IEEE Trans Smart Grid, vol. 5, pp. 1631-1642, 2014.

[13] L. Junqi, T. Junjie, F. Ponci, A. Monti, C. Muscas, and P. A. Pegoraro, "Trade-offs in PMU deployment for state estimation in active distribution grids," IEEE Trans Smart Grid, vol. 3, pp. 915-924, 2012

[14] F. C. Schweppe and R. D. Masiello, "A tracking static state estimator," IEEE Trans Power Appar. Syst., vol. PAS-90, pp. 1025-1033, 1971.

[15] A. S. Debs and R. Larson, "A dynamic estimator for tracking the state of a power system," IEEE Trans. Power Appar. Syst., vol. PAS-89, pp. 1670-1678, 1970.

[16] A. M. Leite da Silva, M. B. Do Coutto Filho, and J. F. de Queiroz, "State forecasting in electric power systems," Proc. IET Gener. Trans. and Distri., vol. 130 pp. 237-244, 1983.

[17] G. Valverde and V. Terzija, "Unscented kalman filter for power system dynamic state estimation," IET Gener. Trans. and Distri. , vol. 5, pp. 29-37, 2011.

[18] M. Brown and J. C. S. Souza, "Forecasting-aided state estimation-part I: panorama," IEEE Trans. Power Syst., vol. 24, pp. 1667-1677, 2009.

[19] G. Durgaprasad and S. S. Thakur, "Robust dynamic state estimation of power systems based on M-Estimation and realistic modeling of system dynamics," IEEE Trans. Power Syst., vol. 13, pp. 1331-1336, 1998.

[20] A. Farzanehrafat and N. R. Watson, "Power quality state estimator for smart distribution grids," IEEE Trans Power Syst., vol. 28, pp. 2183-2191, 2013.

[21] S. Deshmukh, B. Natarajan, and A. Pahwa, "State estimation and voltage/VAR control in distribution network with intermittent measurements," IEEE Trans Smart Grid, vol. 5, pp. 200-209, 2014.

[22] H. L. Liao, Z. X. Liu, J. V. Milanović, and N. C. Woolley, "Optimisation framework for development of cost-effective monitoring in distribution networks " IET Gen. Trans. Distri. (in press), 2015.

[23] Z. Liu and J. V. Milanović, "Probabilistic estimation of voltage unbalance in MV distribution networks with unbalanced load," IEEE Trans. Power Del., vol. 30, pp. 693-703, 2015.

[24] N. C. Woolley and J. V. Milanović, "Statistical estimation of the source and level of voltage unbalance in distribution networks," IEEE Trans. Power Del., vol. 27, pp. 1450-1460, 2012

[25] A. P. S. Meliopoulos, Z. Fan, and S. Zelingher, "Power system harmonic state estimation," IEEE Trans Power Del., vol. 9, pp. 1701-1709, 1994.
[26] Z. Yiquan, X. Yonghai, and X. Yuanyuan, "Research on power system harmonic state estimation," in Proc. Int. Conf. Electric Utility Dereg. and Restr. and Power Tech., 2011, pp. 596-600.

[27] W. Bin, X. Wilsun, and P. Zhencun, "Voltage sag state estimation for power distribution systems," IEEE Trans Power Syst., vol. 20, pp. 806-812, 2005.

[28] M. T. Aung and J. V. Milanović, "Stochastic prediction of voltage sags by considering the probability of the failure of the protection system," IEEE Trans Power Del., vol. 21, pp. 322-329, 2006.

[29] H. L. Liao, S. Abdelrahman, Y. Guo, and J. V. Milanović, "Identification of weak areas of power network based on exposure to voltage sags-Part II: assessment of network performance using sag severity index," IEEE Trans. Power Del. (in press), 2014.

[30] N. R. Watson and K. K. C. Yu, "Transient state estimation," in Proc. Int. Conf. Harm. . Quality of Power, 2008, pp. 1-6.

[31] K. K. C. Yu and N. R. Watson, "Identification of fault locations using transient state estimation," in Proc. Int. Power System Transient, Montreal, QC, Canada, 2005 , pp. 1-6.

[32] I. Roytelman and S. M. Shahidehpour, "State estimation for electric power distribution systems in quasi real-time conditions," IEEE Trans Power Deli., vol. 8, pp. 2009-2015, 1993.

[33] K. A. Clements, "The impact of pseudo-measurements on state estimator accuracy," in Proc. Power and Energy Soc. General Meeting, 2011, pp. 1-4.

[34] A. Angioni, C. Muscas, S. Sulis, F. Ponci, and A. Monti, "Impact of heterogeneous measurements in the state estimation of unbalanced distribution networks," in Proc. Int. Instrum. Meas. Technol.Conf., 2013, pp. 935-939.

[35] C. Muscas, S. Sulis, A. Angioni, F. Ponci, and A. Monti, "Impact of different uncertainty sources on a three-phase state estimator for distribution networks," IEEE Trans. Instru. and Measure., vol. 63, pp. 2200-2209, 2014.

[36] L. Ke, "State estimation for power distribution system and measurement impacts," IEEE Trans Power Syst., vol. 11, pp. 911-916, 1996.

[37] D. Macii, G. Barchi, and D. Petri, "Uncertainty sensitivity analysis of WLS-based grid state estimators," in Proc. Int. Workshop on Applied Measure. for Power Syst., 2014, pp. 1-6.

[38] G. D'Antona and M. Davoudi, "Effects of parameter and measurement uncertainties on the power system WLS state estimation," in Proc. Instrum. Measure. Tech.y Conf., 2012, pp. 1015-1020.

[39] C. Muscas, F. Pilo, G. Pisano, and S. Sulis, "Considering the uncertainty on the network parameters in the optimal planning of measurement systems for Distribution State Estimation," in Proc. Instrum. Measu. Tech. Conf., 2007, pp. 1-6.

[40] J. Pereira, J. T. Saraiva, and V. Miranda, "An integrated load allocation/state estimation approach for distribution networks," in Proc. Int. Conf. Prob. Methods Applied to Power Syst., 2004, pp. 180-185.

[41] R. Singh, E. Manitsas, B. C. Pal, and G. Strbac, "A Recursive Bayesian Approach for Identification of Network Configuration Changes in Distribution System State Estimation," IEEE Trans Power Syst., vol. 25, pp. 1329-1336, 2010.

[42] C. Muscas, M. Pau, P. A. Pegoraro, and S. Sulis, "Impact of input data correlation on Distribution System State Estimation," in Proc. Int. Work. on Applied Measure. Power Syst., 2013, pp. 114-119.

[43] Y. Chakhchoukh, V. Vittal, and G. T. Heydt, "PMU based state estimation by integrating correlation," IEEE Trans Power Syst., vol. 29, pp. 617-626, 2014.

[44] E. Manitsas, R. Singh, B. C. Pal, and G. Strbac, "Distribution system state estimation using an artificial neural network approach for pseudo measurement modeling," IEEE Trans Power Syst., vol. 27, pp. 1888-1896, 2012.

[45] W. Jianzhong, H. Yan, and N. Jenkins, "A robust state estimator for medium voltage distribution networks," IEEE Trans Power Syst., vol. 28, pp. 1008-1016, 2013

[46] I. Dzafic, M. Gilles, R. A. Jabr, B. C. Pal, and S. Henselmeyer, "Real time estimation of loads in radial and unsymmetrical three-phase distribution networks," IEEE Trans Power Syst., vol. 28, pp. 4839-4848, 2013.

[47] Y. Yue and S. Roy, "PMU placement for optimal three-phase state estimation performance," in Proc. IEEE In. Conf. Smart Grid Comm., 2013, pp. 342-347.

[48] P. N. P. Barbeiro, H. Teixeira, J. Krstulovic, J. Pereira, and F. J. Soares, "Exploiting autoencoders for three-phase state estimation in unbalanced distributions grids," Electric Power Systems Research, vol. 123, pp. 108-118, 2015.

[49] S. Nanchian, A. Majumdar, and B. C. Pal, "Three-phase state estimation using hybrid particle swarm optimization," IEEE Trans Smart Grid, vol. PP, pp. 1-1, 2015

[50] M. Nordman, M. Lehtonen, "Distributed agent-based State estimation for electrical distribution networks," IEEE Trans Power Syst., vol. 20, pp. 652-658, 2005.

[51] V. Kekatos and G. B. Giannakis, "Distributed robust power system state estimation," IEEE Trans Power Syst., vol. 28, pp. 1617-1626, 2013.

[52] A. Gomez-Exposito, A. Abur, A. de la Villa Jaen, and C. Gomez-Quiles, "A Multilevel State Estimation Paradigm for Smart Grids," Proc. IEEE, vol. 99, pp. 952-976, 2011.

[53] S. Kuang-Rong and H. Shyh-Jier, "Application of a robust algorithm for dynamic state estimation of a power system," IEEE Trans Power Syst., vol. 17, pp. 141-147, 2002

[54] J. Arrillaga, N. R. Watson, and S. Chen, Power System Quality Assessment. Chichester: Wiley, 2000. 